\title{
Die Fischreste aus den bituminösen Schiefern von Ibando bei Bata (Spanisch Guinea) von Wilhelm Weiler, Worms
}

\author{
Mit 5 Textfiguren
}

Die hier beschriebenen Fischreste sind ein Teil der palaeontologischen Ausbente der letzten Kamerunexpedition des Deutschen Reiches unter Leitung des verstorbenen Dr. ElabeRT 1913-15.

Vom Kriege überrascht, sah sich der Leiter schließlich gezwungen, die südliche Grenze Kameruns zu überschreiten, um neutrales Gebiet, die spanische Kolonie NiederGuinea, zu betreten. Hier setzte Dr. ELBERT seine geologischen Untersuchungen fort und entdeckte bei der Gelegenheit in der Umgebung von Ibando anf dem Nordufer des Rio San Benito (Ejo-Mündung) eine reiche Fischfauna. Durch die Bemühungen von Frau Dr. ElbBerT, welche die Expedition mitmachte, kamen die Fischreste nach dem Tode ihres Gatten in den Besitz der Preubischen Geologischen Landesanstalt, die sie mir Ende Sommer 1921 zur Bearbeitung anvertraute.

Ich danke auch an dieser Stelle allen denen, die mich bei meiner Arbeit unterstiutzten, vor allem Frau Dr. ElBERT-Frankfurt a. M., welche mir einige Tagebuchblätter und eine größere Kartenskizze aus dem Nachlaß ihres Gatten zusandte, und Herrn Prof. Dr. Fr. JAEGER-Berlin, der mir die Tagebücher und den vorläufigen geologischen Bericht ELBERTs zur Einsicht überlieb. Nicht minder verpflichtet fühle ich mich Herrn Geheimrat HerNCKE-Helgoland für seine freundlichen Mitteilungen über einige von mir als mutmaßliche Wachstumserscheinungen bezeichnete Merkmale am Opercularapparat von Leptosomus aethiopicus und Herrn Prof. Dr. HAUPT-Darmstadt für einige Bemerkungen mineralogischer Natur.

Die fischführenden Schiefer sind dunkle Tonschiefer mit dünnen Braunkohlenschmitzen. Sie zeichnen sich durch reichen Gehalt an Bitumen aus, der ihnen eine dunkelgraue bis schwärzliche Farbe verleiht. Sie brennen zunächst mit helleuchtender, stark rußender Flamme, um schließlich nur noch zu g]ühen. Der trockenen Destillation unterworfen, liefern sie ein weikes his gelblichbraunes Gas, das einen teerartigen Geruch besitzt und mit beller, rußender Flamme brennt. Außerdem schlägt sich an den kühleren Teilen der Retortenwand eine dunkelbraune Teerflïssigkeit nieder.

Auf einer ganzen Reihe von Schieferplatten konnten fleckenartig auftretende, dünne, glänzend schwarze Überzüge festgestellt werden, die in einem Falle (Platte $D_{\mathbf{8}}$ ) eine bis zu $2 \mathrm{~cm}$ dicke Schicht bilden. Auch isoliert liegt eine ganze Menge derartiger Brocken vor, wovon einige schon bei schwächerer mikroskopischer Vergrößerung eine feine Längsund eine undeutliche, senkrecht zur ersteren verlaufende Querstreifung aufweisen. Die Substanz ist vollkommen geruchlos und sowohl in Benzin als auch in Petroleum un- 
Wilhelm. Weiler, Die Fischreste aus den bituminösen Schiefern usw. 149

löslich. Beim Erhitzen auf einem Blech bläht sich die Masse auf, wird teigig und entwickelt lebhaft brennende Gase. Als Rückstand bleibt eine koksähnliche Masse, die nur schwer weiter zu oxydieren ist und eine leichte, flockige Asche von schmutziggrauer Farbe hinterläBt. Nach dem Ergebnis der geschilderten Untersuchungen handelt es sich bei der genannten Substanz zweifellos um Gagat.

Weiterhin weisen die Platten z. T. kleine Pyritkristalle auf und manche von ihnen sind geradezn übersät mit Kieselgallen, die wie kleine Hutpilze aus der Oberfläche herausragen.

Auf diesen Sehieferplatten liegen zahlreiche Fischüberreste, die oft in so groBer Zahl vorhanden sind, daB sie sich gegenseitig überlagern. Wohl kaum wird man eine Platte spalten, ohne dab man auf den Spaltflächen neue, meist gut erhaltene Abdriicke findet. An sonstigen organischen Bildungen wurden nur noch ein kleiner wurmröhrenartiger Überrest (Rückseite der Platte $B$ 25) und zahlreiche Ostracodenschalen beobachtet (Platten $D 6$ und 7 ).

Auch die schiefrigen Braunkohlenschmitzen enthalten reichlich Fischüberreste. Selbst unter dem Mikroskop lassen sie keine bestimmbaren Pflanzenteile erkennen, ein Beweis dafür, dah sie aus stark zerstörtem Material hervorgegangen sind. Nur in einem Falle gelang es, auf Platte $D 3$ einen verkohlten Überrest blopaulegen, der sehr walrrscheinlich ein kleines Ästchen vorstellt. Bemerkenswert ist weiterhin noch die Tatsache, daß die Prüfung eines wässerigen Auszuges unserer Kohle auf Jod negativ ausfiel.

Im folgenden geben wir nun die Beschreibung der Fischreste.

\section{Chirocentrites : guinensis nov. sp.}

Fig. 1

Von dieser Art liegen fünf Platten mit Abdrücken vor, wovon drei sich derart lückenlos aneinander fügen, daß sie den ganzen Fisch mit Ausnahme des verloren gegangenen Schädels vorstellen (Platte $C_{1}$ ). Die beiden übrigen enthalten den unvollständigeren Abdruck der anderen Seite (Platte $\mathrm{C}_{2}$ ).

Quer über die Basis der Schwanzflosse unseres Fisches liegt ein Stück aus dem Schwanzabschnitt eines zweiten Exemplares, sehr wahrscheinlich derselben Spezies angehörend, das eine gut erhaltene Analis und Spuren der Dorsalis erkennen läBt.

Das als Type aufgefaßte Exemplar hat eine Wirbelsäulenlänge von $30 \mathrm{~cm}$. Die größte Höhe, zwischen Brustflossen und den Ventralia, beträgt $8,5 \mathrm{~cm}$.

Die leicht wellenförmig gekrümmte Wirbelsäule setzt sich aus 48 ? $(23+25)$ erkennbaren Wirheln zusammen, die aber nur zum Teil noch erhalten sind. Sie waren relativ kurz, ungefähr so breit als hoch und nur gegen das Ende der Wirbelsäule tritt eine leichte Verlängerung ein. Bei allen trägt die Außenseite zwei durch eine Scheidewand getrennte Längsgruben. Die oberen Dornfortsätze sitzen der vorderen Wirbelhälfte auf und erreichen in der Rumpfregion beinahe die Rückenlinie. 
Leider ist in der genannten Gegend bei allen die Basis durch das Gewirr zahlreicher Gräten verdeckt, die erst mit Beginn des Schwanzabschnittes verschwinden. Im allgemeinen sind die Dornfortsätze der Neuralbögen in der Rumpfgegend nur wenig nach hinten geneigt, doch nimmt ihre Neigung zu, je weiter rückwärts sie stehen. Die letzten liegen fast der Wirbelsäule an, indem sie sich gleichzeitig verlängern, um die äußeren Strahlen der Schwanzflosse zu stützen. Die Dornfortsätze der Hämapophysen gleichen den entsprechenden neuralen.

Alle Rippen sind leicht gebogen und erreichen den Bauchkiel, mit Ausnahme der etwas kürzeren letzten. Charakteristisch ist für sie eine Furche, welche sie der Länge nach durchzieht und wie geteilt erscheinen läßt.

Die Brustflosse ist verhältnismäßig gut erhalten und seitlich angeheftet. Über ihr liegt der Abdruck eines breiten kräftigen Knochens,

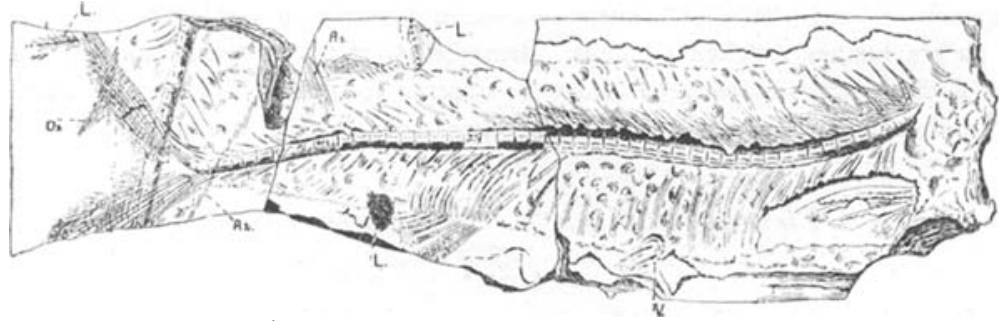

Fig. 1. Chirocentrites? guinensis, n. sp., $1 / 4$ nat. Gr. $-V$ Ventralia; $L$ Überreste von Leptosomus aethiopicus; $A_{2}$ u. $D_{2}$ Analis bezw. Dorsalis des zweiten Exemplars von Ch.? guinensis. - Platte $C_{1}$.

desseu untere Kante nach vorn umgebogen ist. Möglicherweise handelt es sich ·um einen T'eil des Schultergürtels. Die Flosse selbst läßt die Überreste von mindestens sieben Strahlen erkennen, die durch ihre Stärke auffallen. Die ersten drei haben einen starken Außenrand, flachen sich dann zu distalwärts immer breiter werdenden dünnen Platten ab, die sich am Ende schließlich auf das allerfeinste zerteilen. Es scheint, daß beim letzten Strahl diese Verzweigung bereits ganz dicht an der Basis einsetzt.

Die Bauchflossen liegen der Analis ziemlich viel näher als den Pectoralia und zwar unter dem siebenten Rumpfwirbel (von rückwärts gezählt). Infolge des schlechten Erhaltungszustandes kann man keine genauen Angaben über sie machen. Im allgemeinen sind sie im Gegensatz zu den Brustflossen sehr schmächtig, und nur der erste Strahl war vermutlich von einer gewissen Stärke, da er in beiden Flossen einen verhältnismäßig tiefen Eindruck hinterlassen hat. 
Die Rückenflosse beginnt über dem Anfang des 11. (10?) Schwanzwirbels und wird von kleinen, oben breiten, unten zugespitzten Trägern gestützt. Von den letztgenannten sind acht erkennbar. Die Anzahl der schlecht erhaltenen Strahlen beträgt ungefähr zehn. An den größeren tritt bereits dicht über der Basis eine Aufspaltung ein, die bei gleichzeitiger Gliederung nach oben zu immer feiner wird. Bemerkenswert ist, $d a B$ die Glieder durch eine Naht miteinander verbunden sind, die schief zur Längsrichtung verläuft.

Die Analis beginnt nicht gauz sechs Wirbellängen vor der Dorsalis und endigt ungefähr unter dem 6. letzten Schwanzwirbel. Ihre Träger, von denen gegen 30 ermittelt wurden, sind lang, vor allem der erste, welcher zugleich der stärkste unter allen ist. Im allgemeinen schieben sich immer zwei Träger zwischen zwei hämale Dornfortsätze. Die Zahl der Flossenstrahlen läBt sich nur annähernd ermitteln, da die Analis auf eine kurze Strecke durch den Schädel eines Exemplares von Leptosomus aethiopicus verdeckt wird. Sicher waren es ïber 30 . Die ersten 4 oder 5 stehen dichtgedrängt nebeneinander. Zwar fehlt ihr distales Ende, aber immerhin läßt sich auch so erkennen, daß sie die folgenden an Länge beträchtlich überragten.

An der tief ausgeschnittenen Schwanzfosse fehlen ebenfalls die Endabschnitte der beiden Lappen. Jede Hälfte weist $4-5$ stufenförmig größer werdende Randstrahlen auf, wovon nur der längste terminal gegliedert ist. Dazu kommen noch ungefähr 10 Hauptstrahlen, die sich alle fein zerteilen und in derselben Weise gegliedert sind, wie es für die Dorsalis bereits geschildert wurde.

Von Schuppen liegen nur spärliche Überreste vor, die anzeigen, daß sie verhältnismäßig groß, zykloid und konzentrisch gestreift waren. Insgesamt lassen sie ungefähr 4 Längsreihen über bezw. unter der Wirbelsäule erkennen.

Die systematische Stellung des beschriebenen Fisches ist sehr schwer genau zu ermitteln, da seine osteologischen Merkmale auf Beziehungen zu zwei Familien hin weisen: den Leptolepiden mit der Gattung Thrissops einerseits, den Chirocentriden andererseits. Wenn er trotzdem zur letztgenannten Familie gestellt wurde, so geschah es aus folgenden Gründen: die Chirocentriden sind nämlich neben anderen Merkmalen (schräge Stellung der Mundspalte, kräftige Ausbildung des Unterkiefers) noch durch die außerordentlich kräftige Entwicklung der vorderen Pectoralstrahlen charakterisiert. Nun zeigt ja auch Thrissops formosus (1, IV, 2, S. 124, Taf. 65a), die Type dieser Gattung, eine relativ gut entwickelte Brustflosse mit ziemlich breiten ersten Strahlen, die aber, 
was ihre Stärke betrifft, durchaus hinter jenen unseres Exemplares zurückbleiben. Die Art aus den Ibando-Schiefern steht also infolge ihrer spezialisierteren Brustflosse den Chirocentriden näher als Thrissops, so daß eine Einreihung in die erstgenannte Familie als berechtigt erscheint.

Lnter den zahlreichen Gattungen der Chirocentriden stimmt unsere Art am meisten überein mit Chirocentrites HEckeL, sowohl was Stellung, Form und Entwicklung der Flossen, als auch den ganzen Habitus betrifft, so daß eine Einreihung in diese Gattung auf keinerlei Schwierigkeiten stößt. Wenn trotzdem noch ein Fragezeichen beigefügt wurde, so geschah das nur im Hinblick auf die vielen unvollkommen bekannten Chirocentriden-Gattungen, die zum Vergleich gar nicht herangezogen werden konnten.

Von Chirocentrites kennt man bis jetzt nur die Type Chirocentrites coroninii (3, S. 203, Tåf. 13, 14, Fig. 1, 2), Ch. gracilis (3, S. 208, Taf. 15) und Ch. vexillifer (4, S. 245, Taf. 2, Fig. 1,2). Die beiden letztgenannten werden aber von BAssANI zur Gattung Thrissops gestellt (2, S. 257). Von allen dreien unterscheidet sich unsere Art teils durch ihre Größe, teils durch die Stellung der paarigen und unpaarigen Flossen und die Anzahl der Wirbel, so daß wir sie als eine neue Art ansehen müssen, für welche der Name Chirocentrites? guinensis gewählt wurde.

\section{Diplomystus Elberti, nov. sp.}

Fig. 2

Von dieser Art liegen gegen 50, größtenteils gut erhaltene $\mathrm{Ab}$ drücke vor, die sich gegenseitig in der glïcklichsten Weise derartig ergänzen, daß eine Rekonstruktion des Fisches möglich war. Ihr liegt das auch als Type zu betrachtende Exemplar der. Platte $A 6$ zugrunde.

Der Schädel unseres Fisches ist relativ klein und vorn stumpf zugespitzt. Praemaxille und Maxille, hinter denen noch zwei Supramaxillaria liegen, bilden den oberen Rand der Mundspalte. Ihre Form läßt sich aus der Zeichnung leicht erkennen. Alle sind mit dem Schädel am Vorderende fest verbunden, wie aus den Verhältnissen des Exemplares auf Platte $A 1$ hervorgeht, bei welchen das Maul weit geöffnet ist $(8, \mathrm{~S}, 217)$.

Der Opercularapparat ist vollständig. Das von der Anheftungsstelle aus fein gestreifte Operculum ist relativ schmal und an Hinterrand gerundet, das Praeoperculum glatt mit verdicktem Vorderrand. Sein kürzerer horizontaler Ast bildet mit dem längeren aufsteigenden einen Winkel von etwas mehr als $90^{\circ}$. Über die beiden anderen Knochen 
des Kiemendeckels ist nichts Besonderes auszusagen; ihre Form ergibt sich aus der Zeichnung. Von Kiemenstrahlen konnten in einzelnen Fällen $(A 34, A 1)$ Überreste beobachtet werden, die als mäßig gebogene Knochenstäbchen unter dem Vorderende des Praeoperculums hervorragten.

Die Wirbelsäule besteht aus über 30 Wirbeln, wovon 14 auf den kaudalen Abschnitt kommen. Alle sind so lang wie hoch (nur in der Schwanzregion verlängern sie sich leicht) und durch Längsleisten verstärkt. Ihre neuralen Dornfortsätze sind lang und terminal leicht nach rückwärts gekrümmt. Die verlängerten und am Ende abgeplatteten 2-3 letzteu Dornfortsätze helfen die Randstrahlen der Schwanzflosse stützen.

Die langen, leicht gekrïmmten Rippen erreichen nicht ganz den Ventralrand. Während die vorderen unmittelbar an den Wirbeln be-

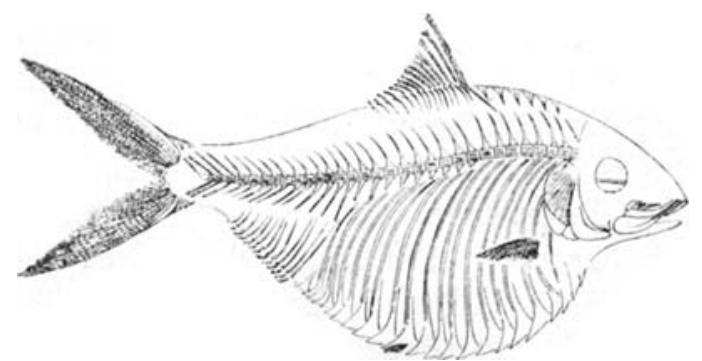

Fig. 2. Diplomystus Elberli, n. sp. Rekonstruiert mit der Type als Grundlage. $1 / 2$ nat. Gr.

festigt zu sein scheinen, sitzen die hinteren sämtlich an kräftigen Wirbelfortsätzen, die nach rïckwärts stetig an Länge zunehmen.

Von den paarigen Flossen sind die Ventralia, unter dem Beginn der zweiten Hälfte der Dorsalis gelegen, sehr klein, geradezu verkümmert und weisen nur wenige (5 ?) Strahlen von geringer Länge auf. Die gut entwickelten Pectoralia mit 14 Strahlen von normaler Länge sind hoch an der Seite angebracht und zwar unmittelbar unter dem Hinterrand des Kienendeckels. Alle Strablen sind gegliedert.

Die Rückenflosse beginnt ungefähr in der Körpermitte (= Mitte zwischen Vorderende und Beginn der Caudalis) und lä3t rund 16 Strahlen erkennen, wovon die beiden ersten ungegliedert sind und auch an Länge hinter dem folgenden gegliederten zurïckbleiben.

Wie die Ventralia, so ist auch die Analis sehr schwach entwickelt. Sie enthält 13 verzweigte und gegliederte Strahlen, deren Kleinheit in einem merkwürdigen Gegensatz zu den langen und kräftigen Trägerno steht. 
Die Caudalis ist sehr kräftig entwickelt und tief eingeschnitten; in jedem Lappen zählt man ungefähr 10 Haupt- und 6 Randstrahlen.

Von den Schuppen finden sich nur einzelne Bruchstücke, die auf eine vollkommen glatte Beschaffenheit der Oberseite hinweisen. In der Ventrallinie sind sie bis zum Beginn der Analis zu sogenannten Kielschuppen umgewandelt, wodurch die Bauchkante wie gesägt erscheint. Sie bestehen aus einem langen aufsteigenden Flügel, der sich nach unten und rückwärts in einen breiten, am Ende scharf zugespitzten Fortsatz auszieht. Ihre Zahl ließ sich nicht ganz genau ermitteln; es mögen ungefähr zwei Dutzend gewesen sein. Auch zwischen Schädel und Dorsalis liegen ungefähr 6 derartig umgewandelter Schuppen. Sie sind länglich dreieckig, am unteren Ende zugespitzt und in der Mitte von einer Längsrippe durchzogen.

Die Gegenwart von dorsalen und ventralen Kielschuppen läßt keinen Zweifel daran aufkommen, daß die vorliegende Clupeiden-Art der Gattung Diplomystus einzureihen ist. Von den zahlreichen Arten, welche von dieser Gattung bis jetzt beschrieben wurden, unterscheidet sich die unsrige deutlich teils durch ibre Größe, teils durch die Form der Kielschuppen oder durch Differenzen in den Körperproportionen, der Stellung der Flossen und ihrer Strahlenzahl. Die beschriebene Form ist eine neue Art, für weiche zu Ehren ihres Entdeckers der Name Diplomystus Elberti in Vorschlag gebracht wird.

\section{Leptosomus aethiopicus, nov. sp.}

Fig. $3-5$

In diesem Falle handelt es sich um ein kleines schlankes Fischchen, von dem rund 50 gute Abdrücke vorliegen, so daß eine Rekonstruktion sich ermöglichen ließ. Als Type gilt das Exemplar der Platte $B \mathbf{5}$, das zwar ein kleineres Individuum vorstellt, aber den vollständigsten Anblick gewährt. Es liegt auch der Rekonstruktion zugrunde. Seine Länge beträgt bis zum Beginn der Schwanzflosse $37 \mathrm{~mm}$, die des Kopfes einschließlich Kiemendeckel $11 \mathrm{~mm}$. Der Kopf ist also in der oben angegebenen Körperlänge rund $3 \frac{1}{2}$ mal enthalten. Die größte Höhe wird etwas vor Beginn der Dorsalis erreicht $(9 \mathrm{~mm})$; sie macht rund $1 / 4$ der Körperlänge aus.

Der Schädel ist ziemlich groß und am Vorderende sehr zugespitzt. Eine genaue Kenntnis der einzelnen Teile des Kiemendeckels gestatten besonders die Exemplare der Platten $B 14, B 16, B 36$ und B 42. Das Operculum ist ziemlich breit, oben etwas schmäler als am Unterrand 
und hinten gerundet. Dicht parallel zum Vorderrand zieht ein kräftiger Wulst, der vom Rande selbst durch eine flache Längsfurche getrennt ist. Die untere Kante ist gerade abgeschnitten und stößt unter einem fast rechten Winkel auf die vordere. Vom Unterrand her strahlen kräftige Rinnen nach dem Anheftungspunkte $\mathrm{zu}$, zwischen die kürzere, aber ebenfalls tiefe Rinnen eingeschaltet sind. Auch am Hinterrand ist diese Zeichnung $\mathrm{zu}$ beobachten, sie verschwindet aber nach oben zu allmählich.

Das Praeoperculum ist relativ schmal und an der Umbiegungsstelle des horizontalen Astes in den vertikalen nur wenig verbreitert. Seine beiden

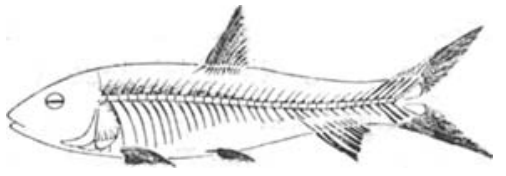

Fig. 3. Rekonstruktion von Leplosomus aethiopicus, n. sp. Nat. Gr. Äste sind gleich lang und stehen senkrecht aufeinander. Sub- und Interoperculum weisen keinerlei Sonderheiten auf.

In einigen Fällen konnten auf Operculum (I und II auf Platte $B$ 18, $B 26$ und $B 24$ ) und Suboperculum (auf Platte $B 37$, I) konzentrische Streifen beobachtet werden, die eine auffallende Ähnlichkeit mit Zuwachsstreifen haben (Fig. 4, 5). Von besonderem Interesse sind die des Suboperculums, wo die breiten Streifen unvermittelt in schmale übergehen. Bei rezenten Fischen kann diese Erscheinung in derselben Weise vorkommen und hängt dann immer mit einem plötzlichen Nachlassen des Wachstums zusammen. Die Ursache davon kann eine zwei-

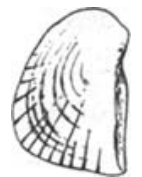

Fig. 4. Isoliertes Operculum von Leptosomus aethiopicus. - Exemplar I, B 49. - Stark vergrößert, von außen gesehen. Mikrosk. Bild.

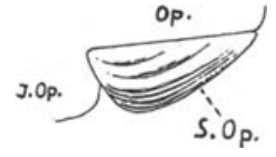

Fig. 5. Leptosomus aethiopicus. Teile des Opercularapparates. $O p$. Operculum; J. Op. Interoperculum; S. $O p$. Suboperculum. - Stark vergr. - Exemplar II, B 37. - Mikrosk. Bild.

fache sein, entweder eine Verschlechterung der äußeren Lebensverhältnisse oder aber die Erreichung eines bestimmten Alters. Aus diesem Grunde bezeichnet HEINCKE auch das plötzliche Schmalerwerden der Jahresringe als den „Beginn des Alterns" (5, S. 172).

Herr Geheimrat HeINCKE, dem ich einige Skizzen mit der Bitte um Beurteilung nach Helgoland sandte, teilte mir mit, daB auch er geneigt 
sei, die Streifen als Wachstumsringe anzusehen, doch könne nur die mikroskopische Untersuchung eines Dünnschliffes darüber einwandfreie Auskunft geben. Leider gelang es aber nicht, die betreffenden Knochen aus dem harten Gestein zu lösen.

Die Wirbelsäule wird von über 30 Wirbeln gebildet, wovon 12 auf den kaudalen und der Rest auf den Rumpfabschnitt kommen. Alle sind leicht verlängert und von schwach sanduhrförmiger Gestalt. Unter dem aufwärts gebogenen verknöcherten Chordarest am Ende der Wirbelsäule erkennt man die Abdrücke von mindestens 2 dreieckig langgestreckten Hypuralplatten.

Die neuralen Dornfortsätze, im vorderen Rumpfabschnitt fast völlig von den zahlreichen Gräten verdeckt, sind dünn, terminal leicht gekrümmt und etwas nach rückwärts geneigt. Die der letzten 5-6 Schwanzwirbel erreichen eine beträchtliche Länge und ihr abgeplattetes Ende dient den Randstrahlen der Schwanzflosse als Stütze. Den nenralen Dornfortsätzen gleichen die entsprechenden hämalen.

Die Rippen beginnen am Wirbelkörper mit breiter Fläche, verschmälern sich aber derart, daß sie schließlich scharf zugespitzt endigen. Sie sind durchweg nur schwach gebogen, erreichen nicht die Bauchkante und nehmen nach rückwärts ziemlich rasch an Länge ab.

Von den unpaaren Flossen liegt die Dorsalis genau in der Mitte zwischen Vorderende und Beginn der Caudalis. Sie enthält zwei ungegliederte und neun gegliederte Strahlen. Der erste Stachel ist bedeutend kleiner als der zweite, welcher seinerseits wieder von dem beinahe doppelt so großen ersten gegliederten Strahl überragt wird. Dieser bleibt nur wenig hinter der größten Höhe des Fisches zurück. Die folgenden acht Strahlen werden ziemlich rasch kleiner.

Die Zahl der Träger beträgt 10, wovon der vorderste die beiden ungegliederten Strahlen trägt und sich von den folgenden deutlich nach vorn zu abspreizt.

Die Analis liegt weit hinter dem Ende der Dorsalis und enthält ungefähr 7-8 Strahlen, die an Länge nur wenig hinter dem vorderstem gegliederten Strahl der Dorsalis zurückbleiben.

Die Candalis ist tief eingeschnitten und weist zwei gleich starke Lappen mit je 5-6 stufenweise größer werdenden Randstrahlen und 10-11 Hauptstrahlen auf.

Von den paarigen Flossen sind die Pectoralia insofern am bester entwickelt, als sie die meisten Strahlen haben: je 10 gegliederte, wozu noch ein 11. wahrscheinlich ungegliederter kommt. Die Ventralia stehen genau in der Mitte zwischen Pectoralia und Anfang der Analis und 
enthalten 7 gegliederte Strahlen und einen kürzeren ungegliederten (Platten $B$ 32, Exemplar II, $B$ 14).

Die Schuppen waren sehr zart. Nur dürftige Überreste sind noch vorhanden, meistens undeutliche Abdrücke mit konzentrischen Streifen. Ü̉ber der Wirbelsäule kann man 5 Längsreihen beobachten.

Der zierlich schlanke Körper, die Zahl der Wirbel und ihre regionale Verteilung, Form und Stellung der paarigen und unpaaren Flossen weisen der beschriebenen Form ihren Platz in der Familie der Scopeliden, Gattung Leptosomus, an. Von diesem Genus sind bis jetzt mehrere Arten aus der Kreide Syriens und Westfalens bekannt geworden. Am meisten Ähnlichkeit hat unsere Art mit L. Guestfalicus (6, S. 50, Taf. 8, Fig. 4, 5) und L. minimus (1, V 2, S. 120, Taf. 61, Fig. 1; 7 , S. $65 ; 9$, IV, S. 246), unterscheidet sich aber von ihnen teils durch Differenzen in den Körperproportionen, der Anzahl der Flossenstrahlen in der Caudalis, teils durch die abweichende Lage der Ventralia. Da diese Art die erste ist, welche auf afrikanischem Boden gefunden wurde, wird für sie die Bezeichnung Leptosomus aethiopicus in Vorschlag gebracht.

\section{Zusammenfassung}

Die in den Ibando-Schiefern festgestellte Fischfauna umfaßt folgende Arten:

1. Chirocentrites? guinensis, nov. sp.

2. Diplomystus Elberti, nov. sp.

3. Leptosomus aethiopicus, nov. sp.

Die Gattung Leptosomus wurde mit ihren Vertretern bis jetzt nur in den marinen Ablagerungen der oberen Kreide Westfalens und Syriens (Libanon, Fundort Hakel und Sahel Alma) nachgewiesen.

Diplomystus beginnt in der unteren Kreide von Istrien und Pietraroja, ist häufig in der oberen Kreide Syriens und Brasiliens, dem Eozän von Wyoming und findet sich auch im Unteroligozän der Insel Wight. In der Gegenwart lebt die Gattung noch fort in den Flüssen von NeuSüdwales und Chile.

Die Chirocentriden, welche augenblicklich nur noch mit einer Gattung und einer einzigen Spezies, dem Chirocentrus dorab des Indischen Ozeans vertreten sind, tauchen mit einer großen Zahl von Gattungen am Ende des Mesozoikums auf. Chirocentrites kommt mit Chirocentrites coroninii in der ältesten Kreide von Istrien vor, während die übrigen Gattungen fast ausschließlich der oberen Kreide angehören. 
Ziehen wir aus diesen Tatsachen den Schluß, so ergibt sich, daß die Ibandoschiefer in die Kreide und zwar, wie Leptosomus aethiopicus anzeigt, mit ziemlicher Bestimmtheit in die obere Kreide zu stellen sind.

Was die Natur der Ablagerungen betrifft, so ist hervorzuheben, daß die fossilen Arten von Diplomystus teils in limnischen, teils in rein marinen Sedimenten vorkommen, während die rezenten, wie bereits mitgeteilt, sich in den Mündungen großer Flüsse aufzuhalten pflegen. Leptosomus und Chirocentrites dagegen wurden bis jetzt nur in marinen $\mathrm{Ab}$ lagerungen gefunden, und wir dürfen darin einen Beweis dafür erblicken, daß die Ibando-Schiefer vermutlich marinen bis brackischen Ursprungs sind.

Auffallend ist nun der Umstand, daß in den allermeisten Fällen die beschriebenen Fossilien oft bis in die feinsten Finzelheiten hinein erhalten sind. Nur bei Leptosomus aethiopicus findet man ab und $\mathrm{zu}$ leicht sich ablösende Knochen, vor allem des Kiemendeckels, etwas verlagert. Die Bildung der Schiefer muß also in einem ganz ruhigen Meeresabschnitt, wahrscheinlich einer stillen Bucht vor sich gegangen sein, die nur eine geringe Tiefe besaß, so daß die abgestorbenen Fische rasch eingebettet wurden.

Von hohem Interesse ist noch der große Reichtum unserer Schiefer an Kohle. Die Pflanzen, aus denen sie hervorging, waren vermutlich nicht marin, wie aus dem Vorhandensein von in Gagat umgewandeltem Holz, sowie aus dem als Zweig gedeuteten Braunkohlenrest hervorgeht. Auch spricht der Mangel an Jod dafür, daB Tange, die als marine Gewächse doch in erster Linie in Betracht kämen, an der Braunkoblenbildung wohl nicht beteiligt waren. Wahrscheinlich haben wir es hier mit Pflanzenresten zu tun, die vom nahen Festland stammten, in der ruhigen Bucht sich ansammelten, teils verkohlten, teils sich zersetzten und so Ursache des reichen Gehaltes an Bitumen wurden, das unsere Schiefer durchsetzt. Daß dabei Schwefelwasserstoff neben anderen Zersetzungsgasen sich bildete, dafür legt der Pyrit-Gehalt des Gesteines Zeugnis ab. Wir haben also in den Ibando-Ablagerungen eine sogenante Faulschlammbildung zu erblicken. Diese Tatsache vermag auch ganz ungezwungen die große Armut tierischen Lebens im allgemeinen und das dazu im Widerspruch stehende massenhafte Vorkommen von Fischüberresten in den Schiefern am besten zu erklären.

Der Umstand nämlich, daß Leptosomus und Diplomystus in großen Mengen häufig dicht neben- und übereinander auf einer Platte liegen, 
deutet darauf hin, daß die Tiere in Schwärmen, ähnlich wie unsere heutigen Heringe zogen ${ }^{1}$ ). Unterstützt wird diese Vermutung noch durch die Tatsache, daß die Fische, nach Aussage ihrer Größe, ungefähr demselben Altersstadium angehören, was bei unseren Heringsschwärmen ja normalerweise auch der Fall ist. Unter 41 vermessenen Exemplaren von Leptosomus aethiopicus war 1 von 28 und 1 von $32 \mathrm{~mm}$ Länge vorhanden. Die übrigen verteilten sich folgendermaßen: $36-40 \mathrm{~mm}=$ 9 Exemplare, $42-45 \mathrm{~mm}=11$ Exemplare, $46-50 \mathrm{~mm}=16$ Exemplare, $52-54 \mathrm{~m}=3$ Exemplare. Die meisten gruppieren sich also um $42-50 \mathrm{~mm}$, eine Differenz, die auf nur geringe Altersschwankung hinweist, so weit sich das überhaupt mit Hilfe dieser Methode bestimmen läßt. Besonderes Augenmerk wurde den Größenverhältnissen von Tieren auf derselben Platte zugewandt, weil wir bei ihnen die meiste Gewißheit dafür haben, daß sie gleichzeitig ums Leben kamen. Mit dem gleichen Erfolg. Auf Platte $B 16$ z. B. beträgt der größte Unterschied zwischen dem kleinsten und größten Exemplar bei 8 meßbaren Individuen nur $6 \mathrm{~mm}$.

Wir gehen daher sicher nicht fehl mit der Annahme, daß die oben genannten Arten sich in Schwärmen dem Lande näherten, vielleicht um zu laichen, und daß dabei, wie es ja auch bei den Heringszïgen heute noch vorkommt, ein Teil in die mehr oder minder vom Meer abgeschlossene seichte Bucht eindrang. Durch die heftigen Bewegungen wurde der Schlamm aufgerührt, und die Zersetzungsgase, welche dadurch in größerer Menge frei wurden, vergifteten und erstickten die Tiere. Bei den schlanken Leptosomiden kann man noch in vielen Fällen an den krampfhaften Verzerrungen und Verbiegungen des Rumpfes die äußeren Anzeichen des Todeskampfes erblicken, während der platt zusammengedrückte Körper der Diplomystus-Arten eine derartige Verkrümmung der Wirbelsäule nicht erlaubte.

Auf eine etwas andere Ursache ist vermutlich die Anwesenheit von Chirocentrites in den Ibando-Schiefern zurückzuführen. Die Angehörigen der Chirocentriden waren, wie aus der sehr kräftigen Bezahnung hervorgeht, ausgesprochene Räuber. Der Umstand, daß Chirocentrites? guinensis gerade mit Schwärmen von Leptosomiden, die kreuz und quer über seinem Abdruck liegen, zugrunde ging, legt den Gedanken nahe, daß er, ähnlich wie z. B. heute Thynnus die Clupeiden, die Leptosomiden und Diplomystus-Schwärme verfolgte, und dabei mit in das todbringende Gewässer hineingeriet.

1) Das schwarmartige Auftreten von Diplomystus wird auch in den Green River Shales und Kreideablagernngen Syriens beobachtet. 
160 Wilhelm Weiler, Die Fischreste aus den bituminösen Schiefern usw.

\section{Literaturverzeichnis}

1. Agassiz 1839/44. Rech. Poiss. foss., Bd. III-V.

2. Bassani 1882. Descrizione Pesci foss. di Lesina. Denkschr. K. Ak. Wiss, math. nat. Cl., Bd. 45.

3. HECKEL 1849. Beitr. zur Kenntnis d. fossilen Fische Österreichs. Denkschr. K. Ak. Wiss., math. natur. Cl., Bd. I.

4. HECKEL 1856. Beiträge zur Kenntnis d. foss. Fische Österreichs. Denkschr. K. Ak. Wiss., math. nat. Cl.. Bd. XI.

5. HEINCKE 1916. Nene Forschungen über Alter und Wachstum der Schollen. Der Fischerbote, Bd. 8.

6. v. D. Marck 1863/65. Fossile Fische, Krebse u. Pflanzen aus dem Plattenkalk d. jüngsten Kreide in Westfalen. Palaeontogr. Bd. XI.

7. Pictet und Humbert 1866. Nouvelles Recherches sur les Poissons fossiles du Mont Liban.

8. Thilo 1920. Das Maulspitzen der Fische. Biol. Zentralbl., Bd. 40.

9. WOODWARD 1901. Catalogue of the fossil fishes etc., Bd. IV. 